Cahiers

d'ethnomusicologie

\section{Cahiers d'ethnomusicologie}

Anciennement Cahiers de musiques traditionnelles

$8 \mid 1995$

Terrains

\title{
Radif. Intégrale de la musique savante persane
}

Dariush Talâ'i, Paris : Al Sur, 1994

Jean During

\section{(2) OpenEdition}

\section{Journals}

Édition électronique

URL : http://journals.openedition.org/ethnomusicologie/1253

ISSN : 2235-7688

Éditeur

ADEM - Ateliers d'ethnomusicologie

\section{Édition imprimée}

Date de publication : 31 décembre 1995

Pagination : 265-269

ISBN : 2-8257-0537-3

ISSN : $1662-372 X$

Référence électronique

Jean During, «Radif. Intégrale de la musique savante persane », Cahiers d'ethnomusicologie [En ligne], 8 | 1995, mis en ligne le 04 janvier 2012, consulté le 21 avril 2019. URL : http:// journals.openedition.org/ethnomusicologie/1253

Ce document a été généré automatiquement le 21 avril 2019

Tous droits réservés 


\title{
Radif. Intégrale de la musique savante
} persane

\author{
Dariush Talâ'i, Paris : Al Sur, 1994
}

Jean During

\section{RÉFÉRENCE}

Radif. Intégrale de la musique savante persane par Dariush Talầi, setâr. Paris : Al Sur, 5 vol. ALCD 116-120, 1994.

1 Précisons d'emblée que le radif de Mirzâ 'Abdollâh (1843-1918), qui est enregistré ici n'est pas, bien entendu, «l'intégrale de la musique savante persane ». D'une part, il existe en effet un certain nombre d'autres radif, d'autre part de nombreuses chansons et pièces instrumentales plus ou moins anciennes ont également un statut canonique, enfin parmi les innombrables pièces classiques que l'on joue, certaines pourront peut-être prétendre à l'avenir à ce statut privilégié.

2 Le radif, qui est le chef-d'œuvre du génie du peuple iranien, est aussi une organisation par laquelle sa musique d'art se distingue de toutes les autres (à l'exception de la tradition de l'Azerbaïdjan qui a élaboré son propre radif - le Muqam - sur des bases communes à celles de l'Iran). Il existe bien en Asie centrale ou en Afrique du Nord des répertoires organisés investis de la même valeur emblématique que le radif, mais celui-ci s'en distingue nettement par sa structure, sa forme hautement originale, son fonctionnement et sa place dans la pratique musicale. Au sens large, le radif (litt. « rang », « rangée », « série ») est un corpus de pièces au rythme en grande majorité non mesuré (ou « élastique »), classées par affinités modales, destinées à être jouées plus ou moins dans un certain ordre et dans la version-modèle d'un grand maître, sous une forme stable ou adaptable.

Il comporte douze systèmes modaux - sept dastgâh, systèmes principaux, et cinq, voire six âvâz ou dérivés - qui englobent environ une trentaine de types modaux (âvâz, shâh gushe) et plus de deux cent types mélodiques ou mélodico-rythmiques (gushe), portant chacune un nom. 
4 Le radif est aussi un modèle exemplaire grâce auquel on apprend: a) le répertoire des mélodies types; b) le classement des modes et des modulations, leur structure, leurs traits typiques ; c) la technique instrumentale, le style classique, les principes esthétiques, les règles implicites de composition. C'est enfin un modèle générateur de formes nouvelles. Dans la pratique, pour certains musiciens, le radif est la quintessence de la musique iranienne et il faut le répéter régulièrement pour ne pas l'oublier; pour d'autres, il est une source d'inspiration et un stock de motifs à exploiter; pour d'autres enfin, il a essentiellement une fonction pédagogique et peut se laisser oublier.

5 Malgré des variantes importantes dans le nombre, la séquentialisation ou la répartition des éléments, tous les radif dénombrent les mêmes types modaux (dastgâh, âvâz, shâh gushe ). C'est donc surtout au niveau de l'actualisation de ces derniers que les différentes sont importantes, voire essentielles d'une école à l'autre ${ }^{1}$.

6 Tout ceci, Talâ'i l'explique en ses propres termes, avec beaucoup d'autres détails, dans les vingt-trois pages du livret français / anglais. On apprend ce qu'il faut savoir sur les intervalles, les échelles, l'instrument, les façons de l'accorder, les concepts clefs tels que mâye (« mode ») - rarement cité par les musicologues, mais essentiel -, dang (tétracordes), etc., l'origine (savante ou populaire) des mélodies, leurs caractères rythmiques (« souple » ou rigide), la fonction du radif, son histoire, etc. On peut lui faire confiance, il connaît son sujet et évite les clichés et les mythes courants.

7 Passons rapidement sur quelques détails (frets pour frettes, Moshtâq Ali Shâh rajeuni d'un siècle), et rectifions, à la demande de Dariush Talâ'i lui-même, deux erreurs dans la notice biographique rédigée par l'éditeur : $a$ ) la thèse citée n'a pas (encore ?) été déposée, et b) le C.E.M.O., association aujourd'hui dissoute, n’a jamais été rattaché à la Sorbonne.

8 Au milieu de ce texte qui «sonne juste ", les mesures d'intervalles que donne Talầi ne manqueront pas de surprendre les connaisseurs, encore que les oreilles fines remarqueront que l'artiste lui-même utilise les intervalles habituels de la musique persane. Ainsi le tétracorde (dang)do, ré, mi, fa, chiffré 200,180, 120 cents ne saurait rendre compte des modes Râst ou Mâhur (dont le demi-ton est quasiment «normal»), à moins de ramener ces modes à leur forme arabe! De même le dang 140, 240, 120 (do, rép, mi-1,5 comma, fa) peut rendre compte du mâye d'Esfahân, mais certes pas de celui de Mokhâlef, Chahârgâh ou Râk. Après enquête, il apparaît que l'intention de l'auteur (qui s'en explique dans la version plus complète qu'il a publiée en Iran) n'est pas de définir les gammes constitutives du radif, mais de remonter aux formes archétypales d'où sont issus les différents tétracordes en usage. Il s'agit donc d'une approche non conventionnelle de la genèse des gammes, qui n'a pas de prétention analytique ou prescriptive. Nous voilà donc rassurés et, de toute façon l'interprétation dément le modèle.

Le radif de Mirzâ 'Abdollâh dont il est ici question fut élaboré à partir des éléments que ce maître recueillit auprès de son oncle Aqâ Gholâm Hoseyn, qui lui-même les tenait de son frère, le prestigieux 'Ali Akbar Farahâni ( $m$. vers 1855), le père de Mirzâ 'Abdollâh, prématurément disparu en pleine gloire, après avoir donné un nouvel éclat à la musique persane. Mirzâ, bien qu'excellent târiste, jouait encore mieux le setâr. Il y a lieu de croire que les airs et le style qu'il transmit sont proches de ceux de 'Ali Akbar. Cependant, malgré l'authenticité incontestable de ce radif, certains experts s'étonnent de sa concision et le soupçonnent de n'être qu'un maigre aperçu de ce qu'était le répertoire avant sa codification. Ils le qualifient de " radif de derrière la porte », allusion au fait que Mirzâ ne l'aurait pas reçu par transmission directe et systématique, mais en écoutant son oncle 
derrière la porte. Selon une autre formule, il ne s'agirait que d'un «radif d'assistant » ( xalife) destiné initialement à un cycle d'enseignement moyen. Ce ne sont là que des suppositions. Par contre tout le monde s'accorde sur le fait que l'interprétation du transmetteur Nur 'Ali Borumand (m. 1976) était un peu sèche, mais que la substance musicale en est exemplaire, et, comme on dit, " propre et nette " (shoste rofte). Ce radif n'a cessé de se répandre entre 1968 et 1976 où N. 'A. Borumand, alors « Titulaire de la chaire de radif » à l'Université de Téhéran, en enseigna oralement une partie et, plus encore, après qu'il l'eut enregistré pour les archives de la Télévision iranienne. En aristocrate éclairé, Borumand avait eu le bon esprit d'occuper ses loisirs en prenant des leçons assidues avec Esmâ'il Qahremâni, qui avait été l'assistant (xalife) de Mirzâ 'Abdollâh, et surtout d'enregistrer le répertoire de ce xalife à une époque où personne ne s'intéressait à ce type de musique. Après la disparition de Qahremâni, il devint donc le dépositaire exclusif du radif censé venir en droite ligne du divin 'Ali Akbar.

Pour la petite histoire, il est intéressant de savoir que, durant des années, l'enregistrement réalisé au târ par Borumand demeura une sorte de secret militaire jalousement abrité dans les coffres de la Télévision. Une raison annexe en était que le maitre interdisait formellement qu'on enregistre ses leçons, sans toutefois empêcher ses élèves de prendre des notes. Par ailleurs, comme pour tous les secrets de ce genre, il y eut des fuites. Le temps était donc venu de penser à une transcription, à laquelle l'auteur de ces lignes s'attela dès 1976, sous les auspices des éditions Sorush de la Télévision Nationale. Prescriptive et non descriptive, cette transcription répond à un souci pratique et note les « doigtés » ou coups de plectre, veillant à séparer les motifs et relier les notes d'une façon pertinente: travail délicat auquel Talâ'i apporta sa contribution. La Révolution arrêta l'ouvrage au moment où il était sous presse. Je pus en dérober l'original avant qu'un autre le fasse, mais des photocopies circulèrent parmi les enseignants et, par la suite, l'une d'elles fut piratée et mise sur le marché avec une préface de M.-R. Lotfi, donnant l'impression qu'il en était l'auteur. Entre temps, les cassettes de Borumand se mirent à circuler discrètement et ce radif devint de plus en plus joué.

Environ dix ans plus tard, les activités culturelles reprirent et je remis le manuscrit sauvegardé au nouveau directeur. La publication suscita l'émoi de certains musiciens et amateurs. L'un d'eux rédigea un article intitulé à peu près « Sommes-nous si empotés que notre radif doive être transcrit par un étranger? » Les notations parurent durant un an sans les cassettes, dont la fabrication traînait; un musicien connu, Hoseyn 'Alizâde, contre-attaqua promptement en publiant l'enregistrement de ce radif avant que ne sortent les cassettes de Borumand. Son interprétation au setâr est, disons-le - c'est le critique musical qui parle ici -, très décevante, et celle de Talâ'i a déjà le mérite de gommer la précédente.

12 Précisons qu'il y eut avant lui d'autres importants dépositaires du radif. Un élève de Mirzâ 'Abdollâh, Montazam al-Hokamâ (Mehdi Solhi) fit, dans les années 1920, une transcription très complète $d u$ radif de son maître. Beaucoup d'éléments se retrouvent dans la publication volumineuse et toujours utile de Musâ Ma'rufi (1962). Les radif de chant furent transmis oralement par Seyyed Ahmad Xân, Jenâb-e Qazvini, 'Ali Xân Nâyeb-e Saltâne (c. 1850-1930), Eqbâl Soltân Azâr (1866-1971), Aqâ Ziâ ol-Zâkerin (m. c. 1960). 'Abdollâh Davâmi (1891-1980) fut le maillon essentiel de la transmission du chant et une version (un peu abrégée) de son radif vocal fut enregistrée par son fidèle disciple Mahmud Karimi et transcrite par M. T. Massoudieh (transcription « descriptive » trop détaillée au gré des musiciens, mais livrée avec les cassettes). 

Qahremâni-Borumand demeure la plus répandue, car c'est avec elle qu'ont été formés les meilleurs joueurs actuels de târ, de setâr, de santur, voire de ney et de kamânche. Ainsi ce radiffinit par éclipser celui de Hoseyn Qoli (autre fils de 'Ali Akbar, m. 1913) uniquement jouable au târ, et transmis oralement durant un demi-siècle par son fils, le grand 'Ali Akbar Shahnâzi avec qui travaillèrent tous les joueurs de târ de l'époque. C'est de lui que Dariush Talâ'i, élève surdoué, apprit le târ en quelques années, avant, dit-il, de « désapprendre » cet instrument lorsqu'il se mit à jouer le radif qu'enseignait Borumand. Fort heureusement, il retomba sur ses pieds et tira parti des deux styles, l'un virtuose, dense, viril et extraverti, l'autre fin, précis et académique, si bien que, très vite, sa propre interprétation transcenda esthétiquement et techniquement le modèle.

A-t-il été pris au jeu et voulait-il donner une grande leçon de setâr? L'écoute de ces disques permettrait de le supposer, cependant, lui-même a expliqué ailleurs comment, jouissant d'une parfaite sérénité d'esprit et d'un environnement naturel exceptionnel dans la baie de Seattle, il a entrepris d'enregistrer ce fameux radif « comme il le sentait ", pour lui-même, en toute pureté et simplicité. Ainsi l'allure tranquille qu'il adopte dans certaines pièces, notamment mesurées, ne correspond pas à un souci pédagogique, mais à un choix esthétique en accord total avec son état d'esprit, son hâl - son mood - du moment. Comme en définitive, c'est le hâl qui authentifie l'interprétation, celle-ci rend aussi bien compte de l'essence du radif que les interprétations plus brillantes, extraverties (majlesi, "publiques») auxquelles il nous a habitués à travers ses concerts ou ses autres enregistrements.

15 Ceci dit, du point de vue du critique, le radif reste le radif, c'est-à-dire une forme académique, une épure, rendue ici de manière claire et précise. Avec elle, les élèves potentiels disposent-ils d'un modèle exemplaire dont l'accès est considérablement facilité par l'indexation des séquences (gushe), au nombre d'une soixantaine par disque. Et pourtant, en dépit des qualités de cette entreprise, il est douteux que les mélomanes partageront l'enthousiasme de certains critiques qui n'ont pas été frappés par la différence entre l'énonciation parfaite du Texte (que Talâ'i, évidemment, joue par cœur) et une interprétation plus libre, telle qu'il en a donné un magnifique exemple au târ dans le Shur qu'il grava pour Harmonia Mundi. Reconnaissons qu'il y a tout de même des parties ou des dastgâh entiers, comme Mâhur, Esfahân ou le Râst-Panjgâh final, où le talent et l'inspiration font oublier la leçon de musique qu'est, par essence, le radif. L'élève et le musicologue y trouveront davantage leur compte que le simple mélomane; mais après tout, et les connaisseurs iraniens l'avouent, certains passages du radif sont ingrats, quoique nécessaires. (C'est pourquoi des maîtres comme Darvish Xân (m. 1924) et Sabâ arrangèrent des versions « légères »d'un abord plus plaisant.) De plus, bien que Mirzâ ait été un grand joueur de setâr, cet instrument n'est peut-être pas le plus approprié pour ce radif qui a été pensé pour le târ et transmis par ce moyen; ainsi les registres aigus (dans les $\hat{a} v \hat{a} z$ ) et certains accordages préconisés (en Segâh par exemple) ne sont pas toujours avantageux pour le setâr. Quoiqu'il en soit, élèves, musicologues et mélomanes avertis ne peuvent que se réjouir de disposer d'une interprétation transparente et élégante de ce joyau auquel toute performance traditionnelle renvoie directement ou indirectement, et qui est comme la pointe du compas sans laquelle le tracé du cercle se perd en divagations.

16 Dariush Talâ'i a publié ces mêmes enregistrements en Iran, suivis d'une nouvelle transcription du radif ${ }^{2}$, qu'il justifie par des arguments pédagogiques : ségmentation des 
motifs afin de faire apparaitre la structure hermétique des gushe, marquage ddes répétitions de notes, etc. L'auteur signe au passage son innovation en inventant trois signes pour remplacer des signes pourtant bien courants et pratiques comme le mordant et le pincé. Il n'est pas évident que la transcription y gagne, mais, quoi qu'il en soit, la tradition, la Parole vivante cristallisée en Texte, exige une herméneutique sans cesse renouvelée afin de livrer son essence.

\section{BIBLIOGRAPHIE}

TALÂ’I Dariush, 1993, Radif-e musiqi-e Irân. 8 cassettes avec livret en persan et en anglais, "A new approach of the theory of Persian Art Music ». Téhéran.

TALÂ’I Dariush, 1995, Radif-e Mirzâ ‘Abdollâh. Notations pédagogiques. Téhéran : Farhang-e mo'aser.

\section{NOTES}

1. Les radif complets les plus répandus sont : - celui deMirzâ 'Abdollâh transmis par E. Qahremâni et N. 'A. Borumand (enregistré ici) et destiné en priorité aux instruments au târ, setâr et santur ; le radif de chant de 'A. Davâmi transmis par M. Karimi, (dont une grande partie fut enregistrée, puis transcrite (Massoudieh, 1978) ; - le radif de târ de Hoseyn Qoli transmis par son fils 'Ali Akbar Shahnâzi ; - le radif de ney dont Hasan Kasâ'i est le dépositaire et qui représente l'école d'Esfahân; - le radif compilé par M. Ma'rufi (1962) qui réunit des éléments épars ; - le radif de chant de Hâtam Asgari Farâhâni (n.1933, élève de Ziâ ol-Zâkerin) tenu caché jusqu'à une date récente par son dépositaire, et qui comporte pas moins de huit cent gushe mais dont l'authenticité et l'originalité ne fait pas encore l'unanimité. L'apparition de ce répertoire monumental remet en question les fondements de l'école dite «moderne» et relativise l'importance et la « vérité » des radif canoniques).

2. Voir à ce propos notre propre transcription dans: Jean During, Le Répertoire modèle de la Musique Persane. Radif de târ et de setâr de Mirzâ 'Abdollâh, Téhéran, Sorush, 1991. (Introduction en français, anglais et persan, notations, 6 cassettes enregistrées). Cet ouvrage n'est distribué qu'en Iran mais l'auteur dispose d'un certain nombre d'exemplaires. 